\title{
Correction to: Treatment of cam-type femoroacetabular impingement using anterolateral mini-open and arthroscopic osteochondroplasty
}

Cheng-Ta Wu', Mohammed Mahameed ${ }^{1}$, Po-Chun Lin ${ }^{1}$, Yu-Der Lu' ${ }^{1}$ Feng-Chih Kuo ${ }^{1}$ and Mel S. Lee ${ }^{1,2^{*}}$

\section{Correction to: J. Orthop Surg Res}

https://doi.org/10.1186/s13018-019-1257-z

In the original publication of this article [1], the first name of the 5th author is wrong.

The correct name should be Feng-Chih Kuo.

The original article has been corrected.

Published online: 08 August 2019

\section{Reference}

1. Wu CT, et al. Treatment of cam-type femoroacetabular impingement using

anterolateral mini-open and arthroscopic osteochondroplasty. J Orthop

Surg Res. 2019;14:222. https://doi.org/10.1186/s13018-019-1257-z.

\footnotetext{
* Correspondence: bone@doctor.com

'Department of Orthopaedic Surgery, Kaohsiung Chang Gung Memorial Hospital, Kaohsiung, Taiwan

${ }^{2}$ Chang Gung University, College of Medicine, 123, Ta Pei Road, Niao Sung District, Kaohsiung, Taiwan
} 